\title{
Effect of Varying Packet Interval Time on Multihop Routing Protocol in Wireless Sensor Network
}

\author{
${ }^{1}$ Namita Sharma and ${ }^{2}$ Parveen Kakkar \\ Dept of CSE, DAV Institute of Engg \& Technology, Jalandhar, India \\ ${ }^{1}$ snamita65@yahoo.com; ${ }^{2}$ parveen.daviet@gmail.com
}

\begin{abstract}
The distinguishing characteristics of sensor networks are that they are basically infrastructure less, self configured wireless networks used to monitor environmental conditions such as temperature, sounds etc. which and communicate with each other using radio signals. The sensor nodes contain non chargeable batteries and they in several rounds of data transmission they soon get drained out of energy. Depending upon the given conditions, the sensor nodes in the random network may be hundreds or thousands in number .Each individual node has its own sensing and computing devices along with the radio transceivers and power components. In this paper, effect of changing the packet interval time on the energy consumption, throughput of the proposed multihop routing protocol is analyzed. It is a substantial fact that change in the packet interval time when the packets are to be transmitted to further levels of hierarchy has a relation with the latency and load balancing of the network. The simulation results show that with the variation of the packet interval time, the throughput attains a peak value at a certain point, then decreases alternately whereas the energy consumed by the protocol remains constant for a particular time interval but the value increases as the packet transmission time interval increases.
\end{abstract}

KEYWORDS:- Packet interval variance, Proposed multihop routing protocol, throughput, energy consumption.

\section{Introduction}

Sensor networks have emerged as a promising tool for monitoring the physical worlds, utilizing selforganizing networks of battery-powered wireless sensors that can sense, process and communicate. They consist of small low power nodes with sensing, Computational and wireless communications capabilities that can be deployed randomly or deterministically in an area from which the users wish to collect data. Typically, wireless sensor networks contain hundreds or thousands of sensor nodes that are generally identical. These sensor nodes have the ability to communicate either among each other or directly to a base station (BS). The sensor network is highly distributed and the nodes are lightweight. Intuitively, a greater number of sensors will enable sensing over a larger area.[1] The main features of WSNs are scalability with respect to the number of nodes in the network, selforganization, self-healing, energy efficiency, a sufficient degree of connectivity among nodes, lowcomplexity, low cost and size of nodes.

As the batteries of the sensor nodes are not chargeable, the need is to make the methods of data transmission so effective that the data should be able to be routed to the intended base station as quickly as possible thus minimizing delays and negating all kinds of the packet drops, routing 
overheads etc along with make the design of the routing protocol energy efficient. In addition to it, WSNs face few other challenges as well. A fundamental challenge to these small networks is that wireless sensor networks are power constrained networks i.e. the wireless sensor node can only be set with a limited power supply usually less than $1.2 \mathrm{~V}$. In some situations, recharge/refill of power resources (battery) might be impossible. So we can say that lifetime of a sensor node is totally dependant on battery lifetime. In a multihop adhoc sensor network, each node plays the double role of data originator and data router. The improper working of a few nodes can cause considerable topological changes and might require rerouting of packets and reorganization of the network. The main task of a sensor node in a sensor field is to detect events, perform quick local data processing, and then transmit the data [2]

The are many applications of WSNs to the real world like environmental monitoring, health care , positioning and tracking, to logistic, localization, and so on but everywhere the longevity of such networks has been a source of concern as parameters such as QoS, maximum data transmission for any ideal network or a specific application cannot be compromised. Once data has been made available to the $\mathrm{CHs}$, the next task is to route that data either using single hop manner or in multihop manner so that it could reach Base station. The results for homogeneous networks are better than heterogeneous networks but this fact is also true that the inclusion of certain heterogeneous nodes in the homogeneous environments can further improve the lifetime of the wireless sensor network. [3] Small periodic data packets are the most common workload in sensor networks, but certain cases arise where larger transfers are needed. Therefore, the larger the packet interval, the more the latency is increased. However, the latency decreases with decreasing packet intervals [4] .Also if the packets are transmitted to the nodes up in the hierarchy by the sensor nodes after certain periodically increased intervals, the network traffic load can be managed effectively although in this process there may be slight increase in the energy consumption of the network but overall efficiency would increase considerably.

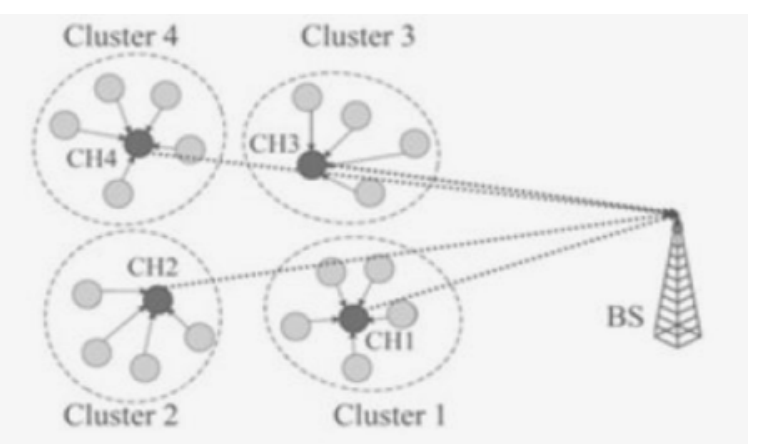

Figure 1: Structure of WSN in LEACH Protocol [5]

The basic structure of Wireless Sensor network consists of normal sensor nodes which are grouped to certain Cluster head nodes which are elected randomly in every round of transmission of data from source to sink. The cluster head nodes are the highest energy nodes in each cluster in a particular round of data transmission. After data aggregation is done by the cluster head nodes, the data is sent to the base station either using flat routing, single hop or multihop routing. But over the years energy efficiency, load balancing, extension of network lifetime many algorithms, techniques and protocols have been developed like LEACH(Low Energy Adaptive Clustering Hierarchy) Protocol [5] followed by HEED[6], A-LEACH[7],C-LEACH[8] etc. The distribution of the paper goes as second section discusses the related work, third section discussed the proposed technique, fourth section 
tells the result analysis and discussion finally the fifth and sixth sections describe the conclusion and future scope of the proposed technique.

\section{Related Work}

Multihop-LEACH can further improved by increasing probability of cluster head and vice cluster head. With a varying probability of clustering, it is clear that more cluster heads in a network results in better connectivity. We can still minimize the energy consumption and extend the network life time by improving the clustering technique[1] The concept of the multihop routing was discussed and implemented in multihop LEACH protocol[9] In this protocol, Multihop-LEACH uses both inter cluster as well as intra cluster communication. The power usage, latency and success rate in Multihop-LEACH can further improved by increasing probability of clustering.Then another multihop routing protocol[10] was proposed which gave the concept of introduction of gateway nodes in the network at the next level to the cluster head nodes. The total number of the gateway nodes was about $10 \%$ of the total number of sensor nodes in the network these would act as intermediaries for the transmission of data from the cluster heads to the base station. They followed a constraint that no two gateway nodes would transfer data to the gateway at the same time, rather the gateway nodes would provide them certain set time slots during which they would transmit data to them. At the given instant of time in the network if one gateway node is not free for transmission of data to the cluster head node, it would not waste time waiting for it turn, rather it would check the availability of other gateway nodes, which so ever is free, it would select it and transfer the data to it for transmission to the base station. Thus with increase of one more hop in the network, there is considerable extension in the network lifetime as compared to the single hop routing protocol.

Yet another protocol named Assisted LEACH[7] focuses on network lifetime goes down when both data aggregation and routing are carried out by Cluster Heads alone which can be eradicated by usage of Helper Nodes for Routing and Cluster Heads for Data Aggregation. It reduced the overhead for route formulation to base station by electing next hop at each Helper Node using the Received Signal Strength values of beckon signal from base station already available at helper nodes during Helper Node Selection phase. The concept of Helper Nodes in Assisted LEACH (A-LEACH) protocol has improved the lifetime of the network by distributing the minimized energy dissipation throughout the nodes.

\subsection{Effect of Packet Interval on Performance of Network}

In a wireless sensor network packet size has the direct effect on reliability and performance of communication between wireless nodes, so there is need to have an optimal packet size for wireless sensor networks. In fact, if a packet transmission fails, the sender has to wait for a random back off period before resuming the packet transmission. However, this period is computed independently from the channel coherence time. Therefore if the channel conditions during retries are still the same or worse, successive failures occur and latency is increased. Network performance would improve if the packet interval depends on the time coherence of the channel. If the interval is too small compared to the coherence time, packet error rate will be high when channel conditions are bad and vice-versa. The packet interval management may also involve the application layer. There is an impact of changing the packet interval on the network performance. The packet transmission time can be tuned in order to optimize the packet delivery ratio. [11]

Sensor networks are predicated using low- power RF transceivers in a multi-hop fashion. Multiple short hops can be more energy-efficient than one single hop over a long range link. Poor cumulative packet delivery performance across multiple hops may degrade performance of data transport and 
Namita Sharma and Parveen Kakkar; Effect of Varying Packet Interval Time on Multihop Routing Protocol in Wireless Sensor Network, Transactions on Networks and Communications, Volume 2 No 6, Dec (2014); pp: 1-11

expend significant energy. The traffic pattern is very simple in wireless sensor network as each node sends roughly $\mathrm{k}$ packets per second with an exponentially distributed inter- packet interval (to avoid synchronization). The traffic load on the network can be adjusted by changing the average load. Periodically, each node broadcasts a packet so that all nodes can construct their neighbor lists. Nodes log received packets in the node's memory [12].

To guarantee the real time performance of the nodes, each data packet is constrained in a time interval in which it must be sent to the destination node. If time expires, the data packet has to be discarded. Once node failure or congestion occurs, large amounts of data packets will be discarded, which may cause disastrous consequences. Consequently, it is more significant and challenging to provide both real-time and fault tolerance characteristics in WSN routing protocol. Failure nodes are treated as an empty area (VOID), and data packets are sent to the sink node via bypass. However, these methods do not predict network congestion in advance, and the remaining transmission time of the data packet is only used for checking the validity of the data packets. When a data packet cannot be transmitted to the next hop node, it will be automatically discarded at once, which wastes the transmission energy. Moreover, the upper stream node cannot receive the feedback information from the current node and thus affect the subsequent transmission. Each node utilizes the remaining transmission time of the data packets and the state of the forwarding candidate node set to dynamically select the next hop. Once node failure, network congestion or void region occurs, the transmission mode will switch to jumping mode, which aims at reducing the transmission time delay and ensuring the data packets to be sent to the destination node within the specified time limit [13].

The sensor network application scenarios and network traffic characteristics differ significantly from conventional computer networks. Typically data is sent periodically in short packets. To achieve fairness and energy efficient transmission through a multihop network, they design an adaptive rate control protocol to that is optimized for n-to-1 data reporting and multihop networking .To let the receiver sleep for most of the time when the channel is idle, nodes periodically wake up and check for activity on the channel. If the channel is idle, the receiver goes back to sleep. Otherwise, the receiver stays on and continues to listen until the packet is received. Packets are sent with long preambles to match the channel check period. Each time the node wakes up, it turns on the radio and checks for activity. If activity is detected, the node powers up and stays awake for the time required to receive the incoming packet. After reception, the node returns to sleep. If no packet is received (a false positive), a timeout forces the node back to sleep. Data that can be delivered by each protocol and the cost of delivering that data. In all tests where we mention "packet size", we are referring to the size of the data payload only, not the header information. [14]

Channel utilization is a traditional metric for MAC protocols that illustrates protocol efficiency. High channel utilization is critical for delivering a large number of packets in a short amount of time .In sensor networks, quickly transferring bulk data typically occurs in network reprogramming or extracting logged sensor data. By minimizing the time to send packets, we can also reduce the network contention.These services implement the appropriate hidden terminal support for their workloads. For example, after sending a multihop message, all nodes in the cell should refrain from transmitting until one packet time has elapsed to allow the parent to retransmit up the tree as proven to be more efficient than control messages for multihop traffic. By allowing the service to decide, many costly control message exchanges are eliminated. Although the network is homogeneous, we can exploit that the base station runs with a different duty cycle (since it is always on) than the data collection network. Instead of sending packets with long preambles, nodes one 
hop away send packets with only an 8 byte preamble to the base station. Note that Lpreamble is reduced from the long LPL preamble to only 8 bytes. The node can return to sleep for the check interval after receiving a packet or can perform early rejection much quicker than packets sent with the long preambles. [14]

\section{Proposed Protocol}

The proposed scheme consists of assumptions, radio propagation model, algorithm which are discussed as:

\subsection{Assumptions of proposed Protocol}

For the proposed protocol [3] some basic assumptions are made which are as under:-

1) These nodes are Mobile and homogeneous in nature.

2) Base station is far away from the network and is fixed.

3) Every sensor node is capable of communicating with every other sensor node scattered randomly in the network and to the Base Station if needed.

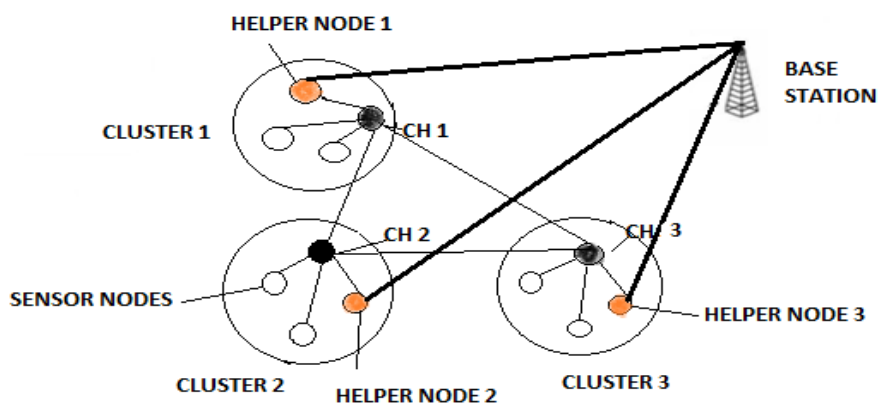

Figure 2: Diagram of the Proposed Scheme [3]

\subsection{Radio Energy Dissipation Model}

For the proposed protocol, the first order radio model is used for energy dissipation in communication [3], where radio dissipates Eelec $=50$ nano Joule $/$ bit to drive the transmitter and the transmit-amplifier dissipates $\varepsilon e l e c=100 \mathrm{pico} J o u l e / \mathrm{bit} / \mathrm{m} 2$. To save energy, when required the radio can be turned on or off. Also the radio spends the minimum energy required to reach the destination. The energy consumed for data transmission of $\mathrm{k}$ bits packet is calculated from the Eq. (1).

$$
\operatorname{ETX}(k, d)=\text { E elec }^{*} k+\varepsilon \text { elec } * k^{*} d 2
$$

and to receive this message, the radio expends energy is shown in Eq. (2):

$$
E \operatorname{Rx}(k)=E \text { RX-elec }(k)
$$

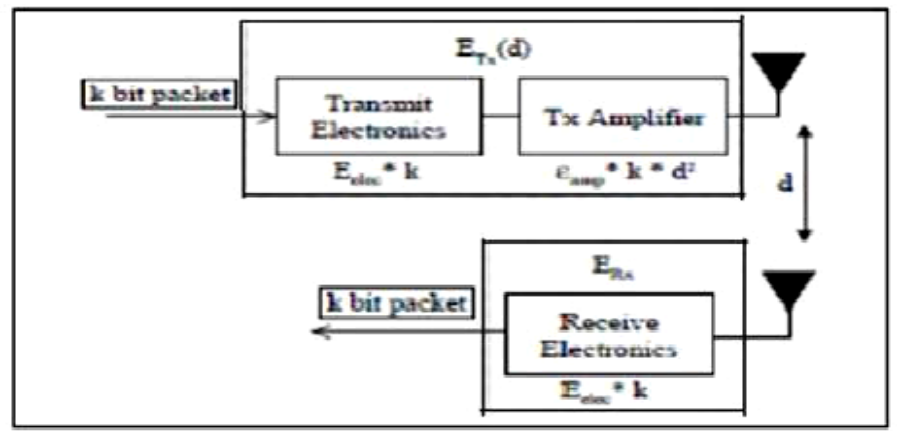

Figure 3: Radio Dissipation Model [3] 


\subsection{Algorithm of Proposed Protocol}

The main goal of the approach is to extend network lifetime of the network [3]. For this reason, cluster head selection is mainly based on the residual energy of each node. The highest energy node that is if the remaining battery power is high then that node will become $\mathrm{CH}$ and the least mobility node will become a $\mathrm{CH}$. Distance of a node from the cluster centroid. The BS calculates the distance of each node to its cluster centroid. The lesser distance node from the BS to itself will have the higher probability to become a $\mathrm{CH}$. The network initialization phase starts after the sensor nodes are randomly distributed in the application area. The base station broadcasts a "HELLO" message to all the nodes in the network to ensure that the network is alive.

The algorithm starts with randomly selects a starting node that has not been visited and it retrieves all neighbor nodes which is density reachable from starting node with respect to Eps and MinPts. Here Eps is a radius of the cluster and MinPts is a minimum nodes required inside the cluster. If the number of neighbors is greater than or equal to MinPts then the cluster is formed as Let the distance between two sets of nodes $\mathrm{S} 1$ and $\mathrm{S} 2$ be defined as dist $(\mathrm{S} 1, \mathrm{~S} 2)=\min \{$ dist $(\mathrm{p}, \mathrm{q}) \mid \mathrm{P} \varepsilon \mathrm{S} 1, \mathrm{q}$ $\varepsilon$ S2 $\}$ and further the nodes with the highest energy are selected as cluster heads by the sensor nodes to which "ADVERTISEMENT" message is broadcasted by the $\mathrm{CH}$ and all the sensor nodes which join the cluster reply back with "ACK" message. The next phase deals with the selection of the cluster heads for each cluster. After the clusters are formed, the Base station should decide whether or not the node becomes a cluster head for the current round. To find that, the value of energy are computed for all the nodes in the network for each round. The node which has highest residual energy is elected the cluster head for the specific round.

Once the clusters are created and the $\mathrm{CH}$ issues a TDMA schedule to all the other sensor nodes in the clusters during which they need to transmit data to their Cluster heads. Base Station constantly observes the residual energy and Mobility of the existing $\mathrm{CH}$. If it is below the threshold value then it select another $\mathrm{CH}$ based on same conditions, described earlier. Finally the $\mathrm{CH}$ should be checked out the routing path. If the routing path residual energy goes below the threshold or any node fails, BS selects another path and sends the routing path to the respective $\mathrm{CH}$. So, the base station calculated the distance of all nodes in the network to itself using RSSI value [3] which is calculated with the help of two ray ground model

$$
P_{r}(d)=\frac{P_{t} * G_{t} * G_{r} * h_{t}^{2} * h_{r}^{2}}{d^{4} L}
$$

Where $P_{r}$ : Power received at distance $d P_{t}$ : Transmitted signal power $G_{t}$ : Transmitter gain (1.0 for all antennas) $G_{r}$ : Receiver gain (1.0 for all antennas) d: Distance from the transmitter L: Path loss (1.0 for all antennas) ht: Transmitter antenna height (1.5 $\mathrm{m}$ for all antennas) hr: Receiver antenna height (1.5 $\mathrm{m}$ for all antennas)

The data aggregated by all the cluster heads are sent to the helper nodes. The helper nodes are those which have second highest energy left in them at the end of each round. Sometimes there might be a situation when there is no such helper node left inside the cluster as it too has been drained out of its energy so in that case the cluster head would search for some other available nearby helper node in some other cluster to which data can be transmitted. The cluster heads enter into sleep mode once they transmit data to the helper nodes so that their energies are saved. At a given time, all the cluster head nodes send data to the helper nodes using multihop routing. Further the helper nodes are informed of the shortest path calculated by the base station along which the 
data is transmitted again by multihop routing[3].Thus this protocol would enhance the performance as well as improve the lifetime of wireless sensor network.

\section{Result Analysis and Discussion}

The simulation scenario consists of 50 sensor nodes deployed in the network field of size $1300 \mathrm{~m} * 1000 \mathrm{~m}$ in the wireless sensor network. All the simulations have been performed using NS2.The results have been obtained at the end of seven rounds of the network at simulation time $=30 \mathrm{sec}$ for both the protocols. The blue line shows the results of the proposed protocol. The main objective of simulation is to analyze the effect on proposed multihop routing protocol by varying packet interval.

Table 1: Simulation Parameters

\begin{tabular}{|c|c|}
\hline Simulator & Ns-2.35 \\
\hline Simulation time & $30 \mathrm{sec}$ \\
\hline Channel Type & Wireless \\
\hline No of nodes & 50 \\
\hline Topology & $1300 \mathrm{~m} * 1000 \mathrm{~m}$ \\
\hline Radio Propagation model & Two way ground \\
\hline Communication Model & Bi direction \\
\hline Transmission Range & $250 \mathrm{~m}$ \\
\hline Interface Queue Type & Queue/Drop Tail/Pri Queue \\
\hline Initial energy & 100 Joules \\
\hline Antenna Type & Omni Antenna \\
\hline Traffic Type & CBR \\
\hline Packet Size & 256 bytes \\
\hline
\end{tabular}

\subsection{Performance Metrics}

The performance analysis of the proposed protocol is done by analyzing the results of Proposed Protocol by using some of the performance metrics such as:

- Throughput: It is the measure of the number of bits of data packets that are transmitted from source to destination in given time. It is always less than 1 . The formula of measuring throughput is

\section{Number of bytes received Time in milliseconds}

Generally it is measured in $\mathrm{Kb} / \mathrm{sec}$ or Bytes/sec. For the protocol aiming to enhance the throughput of the network, it is must that the packet drop rate, jitters, routing overheads and congestion or packet loss should be as less as possible otherwise lower value of throughput would decrease the data packets delivery from the source to the destination.

- Average Energy Consumption (Ea): The average energy consumption is calculated across the entire topology. It measures the average difference between the initial level of energy and the final level of energy that is left in each node.

Let $E_{i}=$ the initial energy level of a node, $E_{f}=$ the final energy level of a node and $N=$ number of nodes in the simulation. Then

$$
E_{a}=\sum_{k=1}^{n}\left(E_{i k}-E_{f k)} / N\right.
$$

This metric is an important because the energy level the network uses is proportional to the network's lifetime. The lower the energy consumption the longer is the network's lifespan. Thus the ideal value for average energy consumed by the protocol should be as less as possible otherwise if 
the protocol would consume more energy after every round then it would become difficult to increase the lifetime of the network. The exact formula for calculation of average energy is inbuilt in NS2.

Here two cases are to be analyzed to understand the effect that the packet interval has on overall efficiency of the protocol by initially setting the packet interval at value of one second and then at three seconds and further understanding the impact on throughput and energy consumption .

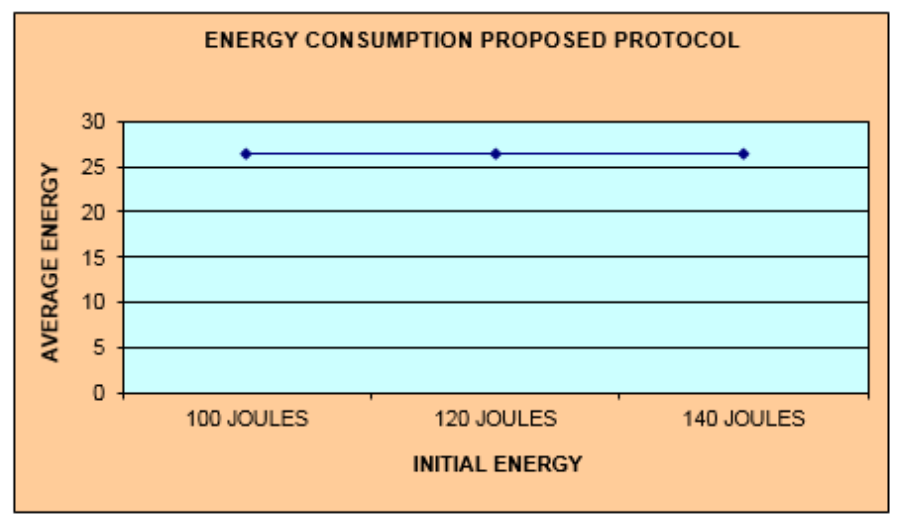

Figure 4: Graph showing Proposed protocol based on energy consumption when packet interval is 1 second.

The graph clearly shows that on running the simulation for seven rounds, when packet size is kept at 256 bytes and the initial energy is varied from 100 Joules to 140 Joules, the average energy consumed by the proposed protocol remains constant at value of 26 Joules. This clearly indicates that with the increase in the packet interval time (transmission), the protocol remains unaffected on the grounds of energy consumption while routing packet from source to sink round by round.

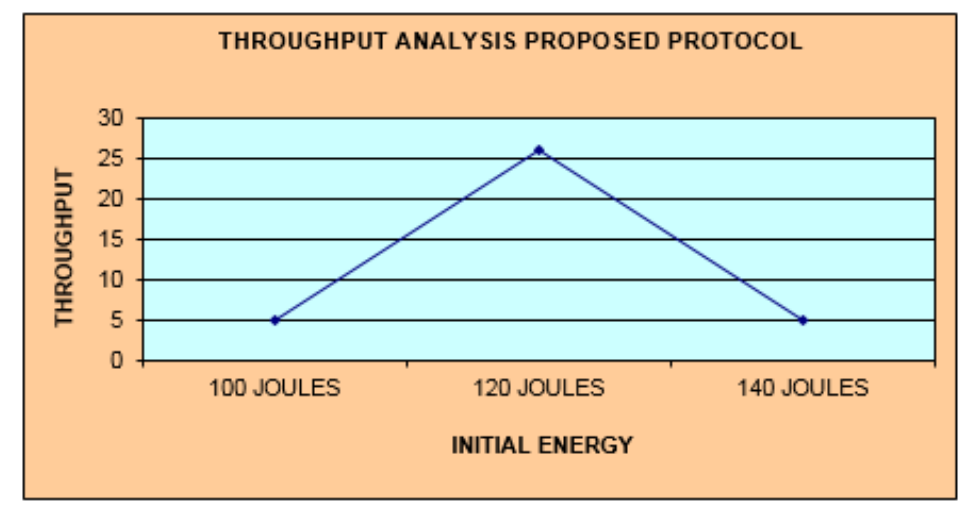

Figure 5: Graph showing Proposed protocol based on throughput when packet interval is 1 second

The graph clearly shows that on running the simulation for seven rounds, when packet size is kept at 256 bytes and the initial energy is varied from 100 Joules to 140 Joules, the throughput of the proposed protocol attains peak value at 120 Joules initial energy and declines for 140 Joules which means that on increasing the value of packet interval the proposed protocol starts consuming more energy although at certain point of time the throughput of the protocol increases and decreases alternately. 


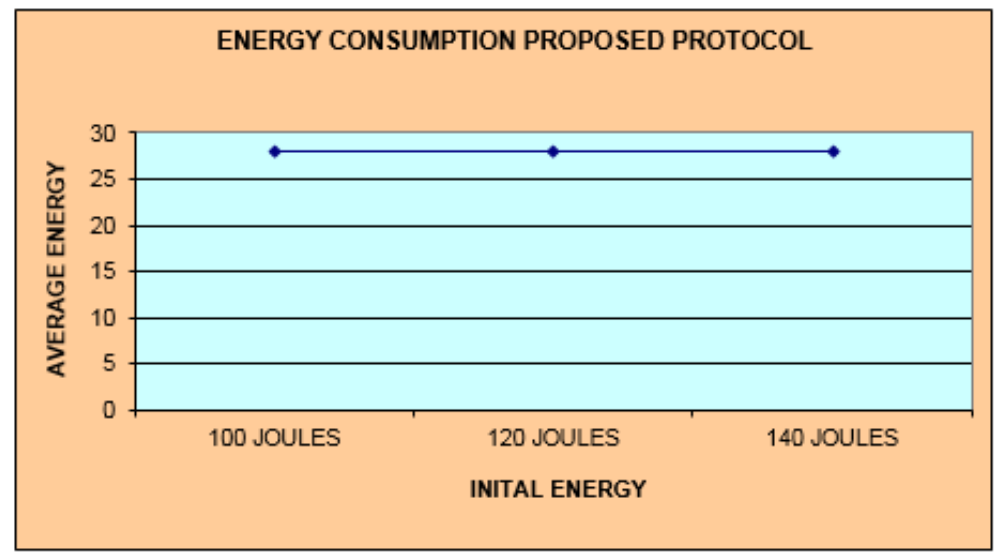

Figure 6: Graph showing proposed protocol based on energy consumption when packet interval is 3 second The graph clearly shows that on running the simulation for seven rounds, when packet size is kept at 256 bytes and the initial energy is varied from 100 Joules to 140 Joules, the average energy consumed by the proposed protocol remains constant at value of 28 Joules. This clearly indicates that with the increase in the packet interval time (transmission), the protocol remains unaffected on the grounds of energy consumption while routing packet from source to sink round by round but the net energy consumption is slightly increased after every interval.

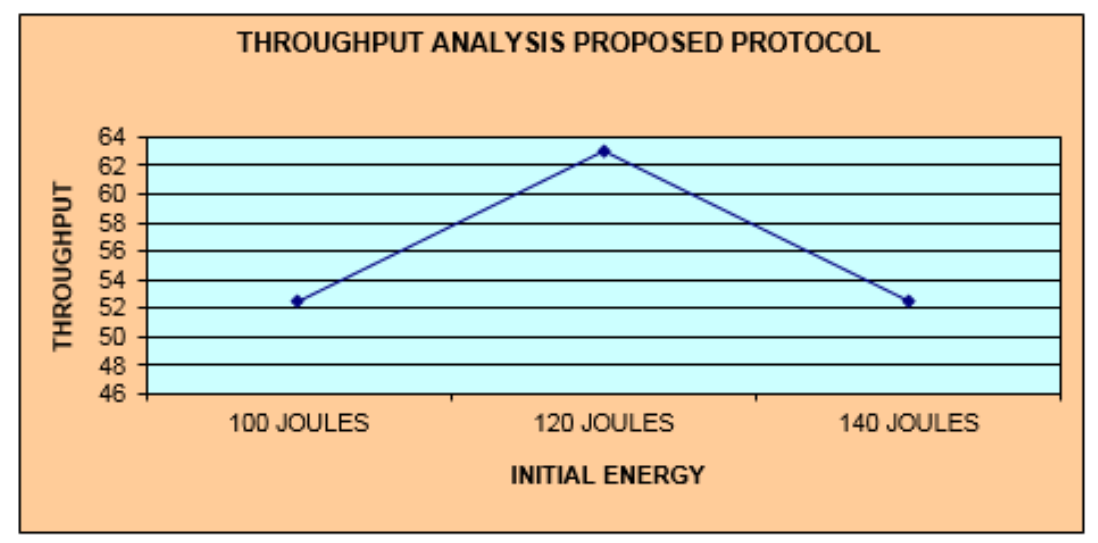

Figure 7: Graph showing proposed protocol based on throughput when packet interval is 3 second

The graph clearly shows that on running the simulation for seven rounds, when packet size is kept at 256 bytes and the initial energy is varied from 100 Joules to 140 Joules, the throughput of the proposed protocol attains peak value at 120 Joules initial energy and declines for 140 Joules which means that on increasing the value of packet interval the proposed protocol start consuming more energy although at certain point of time the throughput of the protocol increases considerably as compared to the value at packet interval time of one second but then decreases afterwards.

Table 2: Summary of Results: PACKET INTERVAL = 1 second

\begin{tabular}{|c|c|c|}
\hline \multirow{2}{*}{ Initial Energy } & \multicolumn{2}{|c|}{ Proposed Scheme } \\
\cline { 2 - 3 } & Throughput & Average Energy \\
\hline 100 Joules & $5 \mathrm{~Kb} / \mathrm{s}$ & 26.5 Joules \\
\hline 120 Joules & $26 \mathrm{~Kb} / \mathrm{s}$ & 26.5 Joules \\
\hline 140 Joules & $5 \mathrm{~Kb} / \mathrm{s}$ & 26.5 Joules \\
\hline
\end{tabular}


Table 3: Summary of results: packet interval = 3 second

\begin{tabular}{|c|c|c|}
\hline \multirow{2}{*}{ Initial Energy } & \multicolumn{2}{|c|}{ Proposed Scheme } \\
\cline { 2 - 3 } & Throughput & Average Energy \\
\hline 100 Joules & $52.5 \mathrm{~Kb} / \mathrm{s}$ & 28 Joules \\
\hline 120 Joules & $63 \mathrm{~Kb} / \mathrm{s}$ & 28 Joules \\
\hline 140 Joules & $52.5 \mathrm{~Kb} / \mathrm{s}$ & 28 Joules \\
\hline
\end{tabular}

\section{Conclusion}

The above results provide an insight to the fact that by keeping the packet size constant and varying the packet interval while transmission of data over the network and analyzing its impact on the various performance metrics like average energy consumption and throughput, the inference thus drawn is that at lower packet interval time, when the data is transmitted to the base station, the protocol expends less energy but as the packet interval time increases, the energy consumption of the proposed protocol increases though it remains constant for a particular time interval and similarly the value of throughput increases and decreases for the proposed protocol at alternate intervals in accordance with the change in initial energy.

\section{Future Scope}

In future the effect on the various other performance metrics can be determined by simulating the proposed protocol on other simulators or by simulating it on other range of applications like VBR (Variable Bit Rate), VOIP etc. Apart from it, the environment can be varied from homogeneous to heterogeneous and its implications can be studied. In addition to it, this protocol can be evaluated by applying to certain specific case studies so that its implications can be interpreted in a wide scenario.

\section{REFERENCES}

[1]. J S Rauthan, S Mishra, An improved Cluster Based Multi-hop Routing in Self-Organizing Wireless Sensor Networks, International Journal of Engineering Research \& Technology (IJERT) Vol. 1 Issue 4, June - 2012 ISSN: 2278-0181

[2]. Mehjabeen leghari,Shazia Abassi,Dr Lachhman Das Dhomeja, Survey on Packet Size optimization techniques in Wireless Sensor Networks, Institute of Information and Communication Technology, University of Singh,Janshoro.

[3]. Namita Sharma, Impact of Varying Packet Size on multihop routing protocol in Wireless Sensor Networks, IJASCSE,Volume 3,Issue 9, 2014.

[4]. Dae-Suk Yoo, Seung Sik Choi, Medium Access Control with Dynamic Frame length in Wireless Senor Networks, Journal of Information Processing Systems,Volume 6, No.4, December 2014.

[5]. Wendi B. Heinzelman, Anantha P. Chandrakasan, and Hari Balakrishnan,An ApplicationSpecific Protocol Architecture for Wireless Microsensor Networks, 660 IEEE Transactions on Wireless Communications, Vol. 1, No. 4, October 2002. 
[6]. Amir Akhavan Kharazian1, Kamal Jamshidi and Mohammad Reza Khayyambashi,Adaptive Clustering in Wireless Sensor Network: considering nodes with lowest energy, International Journal of Ad hoc, Sensor \& Ubiquitous Computing (IJASUC) Vol.3, No.2, April 2012

[7]. Sunkara Vinodh Kumar and Ajit Pal, Assisted-Leach (A-Leach) Energy Efficient Routing Protocol for Wireless Sensor Networks, International Journal of Computer and Communication Engineering, Vol. 2, No. 4, July 2013.

[8]. J.Gnanambigai, Dr.N.Rengarajan, K.Anbukkarasi, Leach and Its Descendant Protocols: A Survey, International Journal of Communication and Computer Technologies Volume 01 - No.3, Issue: 02 September 2012 ISSN Number: 2278-9723

[9]. S.Koteswararao, M.Sailaja, T.Madhu, Implementation of Multi-hop Cluster based Routing Protocol for Wireless Sensor Networks, International Journal of Computer Applications (0975 - 8887) Volume 59- No.8, December 2012

[10]. S. Taruna, Rekha Kumawat, G.N.Purohit, Multi-Hop Clustering Protocol using Gateway Nodes in Wireless Sensor Network International Journal of Wireless \& Mobile Networks (IJWMN), Vol 4, No 4, August 2012.

[11]. Chiraz Chaabane, Alain Pegatoquet, Michel Auguin, Maher Ben Jemaa, A Joint Mobility Management Approach and Data Rate Adaptation Algorithm for IEEE 802.15.4/Zigbee Nodes, Scientific Research, Wireless Sensor Networks, 2014,6,27-34.

[12]. Jerry Zhao, Ramesh Govindan, Understanding Packet Delivery Performance in Dense Wireless Sensor Networks, ACM, SenSys, Nov 5-7,2003.

[13]. Guowei Wu, Chi Lin,Feng Xiq, Lin Yao, He Zhang,Bing Liu, Dynamical Jumping Real -Time FaultTolerant Routing Protocol for Wireless Sensor Networks, School of Electronics \& Information, Dalian University of Technology.

[14]. Joseph Polastre,Jason Hill, David Culler, Versatile Low Power Media Access for Wireless Sensor Networks, SenSys-04, November 3-5, 2004,Baltimore, Maryland,USA. 